\title{
Medical biophysics as a combination of the traditional educational method and e-learning
}

\author{
David Kordek \\ Department of Medical \\ Biophysics, Charles University, \\ Faculty of Medicine in Hradec \\ Kralove, Simkova 870, 500 03, \\ Hradec Kralove, Czech Republic \\ Email: kordekd@lfhk.cuni.cz
}

\author{
Martin Kopecek \\ Department of Medical \\ Biophysics, Charles University, \\ Faculty of Medicine in Hradec \\ Kralove, Simkova 870, 500 03, \\ Hradec Kralove, Czech Republic \\ Email: kopecema@lfhk.cuni.cz
}

\author{
Petr Voda \\ Department of Medical \\ Biophysics, Charles University, \\ Faculty of Medicine in Hradec \\ Kralove, Simkova 870, 500 03, \\ Hradec Kralove, Czech Republic \\ Email: vodap@lfhk.cuni.cz
}

\begin{abstract}
At the Charles University, Faculty of Medicine, in Hradec Kralove, the Biophysics and Biostatics subject is implemented as a traditional combination, i.e. contact, educational method and e-learning. The e-learning is realized in the LMS Moodle in the "multicourses" called Biophysics and Biostatics - General Medicine and Biophysics and Biostatics Dentistry. A process of teaching during the whole term is described in this contribution too. One of the important points is the explanation of the evaluation system of the key activities that are realized in the LMS Moodle. As a part of contribution are added the statistical results of the success rate of all the Online tests to lab and Credit tests. In the conclusion, the reader gets to know our main aim, which is the completion of the student's complex evaluation during the whole "multicourse". This complex evaluation demands the interconnection of the interactive Excel protocols with the LMS Moodle.
\end{abstract}

\section{INTRODUCTION}

A T THE Charles University, Faculty of Medicine, in Hradec Kralove, the Biophysics and Biostatics subject is implemented as a traditional combination, i.e. contact, educational method and e-learning, that is defined in [1]. A number of software tools is used to create e-learning courses as, e.g. WebCT, Blackboard, Adobe Connect, etc. [2]. For about 10 years, e-learning has been implemented using the LMS Moodle software on the Moodle LFHK portal (moodle.lfhk.cuni.cz). Currently, the system Moodle constitutes more than a half of all the installations of LMS (Learning Management System) systems on the world [3]. Our faculty, especially the Department of Medical Biophysics, has many years of experience with e-learning, which is obvious from [4] - [6]. Since the start of this approach, Moodle has been primarily used as a space to upload files to. To a small degree, optional tests for students were implemented; however, they didn't follow a comprehensive concept. Our goal was to gradually find a comprehensive approach on how to evaluate each student's activities in Moodle within the Biophysics and Biostatics subject. Another target was to create e-learning lessons

This work was written with the support of the project of the Ministry of Education MŠMT IP 2016-2018 63 Creating of multi-platform systems for Education support including tools for user friendly support (both mandatory and optional) students could go through without a teacher's assistance. In this way, an extensive textbook on statistics, available at Moodle $L F H K$, was created, as well as e-learning courses used to support practical laboratory tasks. These workshops include, for example, interactive guides for practical exercises combining the scientific and didactic approach to a specific problem. An example is a laboratory task to measure the firmness of a nitinol stent, where the theory of this task is a result of [7] - [9]. In the academic year 2012/13, two new "multicourses" called Biophysics and Biostatics - General Medicine 2012/13 and Biophysics and Biostatics - Dentistry 2012/13 were established for both educational programs. In fact, both courses virtually have the same structure, basic controls, and settings. Therefore, we will focus on the course for general medicine.

\section{METHODS AND MATERIALS}

General medicine students are divided to 5 study groups during the registration process (within each biophysics group, the students are divided into approx. 10 units of 3 members), thereby, a group reporting process was created. This reporting system enables filtering test results and other activities based on the groups. Simultaneously, it enables submitting seminar tasks as a group. All these activities are the key for further implementation. The basic concept was created to enable copying of the above mentioned course, Biophysics and Biostatics - General Medicine 2012/13, at the end of the year, name the copy during the current year, and move the original course to the archive. To enable this, each mandatory activity and material had to be created as a part of this course.

The course is divided into 9 topics. This structure brings an advantage that only the current year changes in several topics and the rest of the information remains the same. The topics of the course include:

1. Information on the subject

2. Lectures for the current year

3. Self-study

4. Practical lessons - labs

5. Practical lessons - statistics 
6. Practical lessons - informatics

7. Practical lessons - test seminars

8. Students' projects

9. Continuous testing

Topics 1 and 2 contain general information only. In topics 3,5 , and 6 , students can use links to existing courses. These courses were designed as separate units - to enable other students, which we do not want to enroll to the above mentioned "multicourse" for the current year, to access these courses. These courses are optional, and potential course grades are not necessarily included in the overview of "multicourse" grades. Topics 4, 8, and 9 contain required and monitored activities, usually linked to the contact education method, i.e. seminars or lectures. Therefore, grades from these activities are included in the grade overview. This concept ensures that all activities necessary to obtain a credit are available in a single integrated grade overview. Topic 4 is the key topic used to combine the traditional educational method and e-learning.

This topic enables students to use links to guidelines for laboratory measurement, again designed as e-learning courses. This way, students can get ready for both theory and measurement before they actually do it. Laboratory measurements are done using the contact educational method in biophysics laboratories. Interactive protocols are another part of topic 4. Each of 5 laboratory measurements has its own output - an interactive protocol the student uses to record their measured values. The protocol also contains Excel macros, which automatically verify the calculations, including statistical tests. Based on this, the student immediately knows whether their calculations are correct or not. Moreover, metadata from the protocol is uploaded to a server after the student finishes their protocol by clicking on Submit. These interactive protocols advantageously provide students with instant feedback. The main benefit for teachers is that protocols are evaluated automatically. The goal is to try to modify the protocols so that a record of the protocol is automatically uploaded to the students' records in Moodle for the above mentioned "multicourse" and a grade is assigned. The third part of topic 4 are Online Tests to Labs (hereinafter only the Online tests). These tests are directly attached to the course; therefore, their evaluation is also available in the general grade overview and can be used to verify whether students are ready to perform a measurement task.

Each of 5 online tests contains 5 questions with a time limit of 10 minutes. The tests are mandatory, and each student must complete a specific test corresponding to a measurement task in the computer classroom before they can do the task. The goal of the test is to clarify how well students are prepared to execute laboratory measurement tasks, both in the theoretical and practical manner. As these tests have been used in this form since the 2012/13 academic year, they provide interesting statistical information for overview. Moreover, they are included in comprehensive student evaluation of the subject, and the evaluation process is fully implemented in the above mentioned "multicourse". As stated above, this complex model was established in the 2012/13 academic year. For results of the average success rate for each of the 5 online tests, see Fig. 1 and Tab. 1.

TABLE I.

AVERAGE SUCCESS RATE [\%] FOR ONLINE TESTS FOR THE LAST 5 YEARS

\begin{tabular}{|l|l|l|l|l|l|}
\hline & Senses & ECG & $\begin{array}{l}\text { Ultra- } \\
\text { sound }\end{array}$ & CT & $\begin{array}{l}\text { Micro- } \\
\text { scopy }\end{array}$ \\
\hline $\mathbf{2 0 1 2} / \mathbf{1 3}$ & 96,5 & 94 & 84,9 & 94,5 & 85,5 \\
\hline $\mathbf{2 0 1 3} / \mathbf{1 4}$ & 62 & 82,6 & 85,9 & 91,5 & 87,3 \\
\hline $\mathbf{2 0 1 4} / \mathbf{1 5}$ & 69,1 & 86,3 & 71,3 & 76,1 & 84,6 \\
\hline $\mathbf{2 0 1 5} / \mathbf{1 6}$ & 67,6 & 87,6 & 69,1 & 75,8 & 84 \\
\hline $\mathbf{2 0 1 6 / 1 7}$ & 62,8 & 87,3 & 63,7 & 72,9 & 89,9 \\
\hline
\end{tabular}

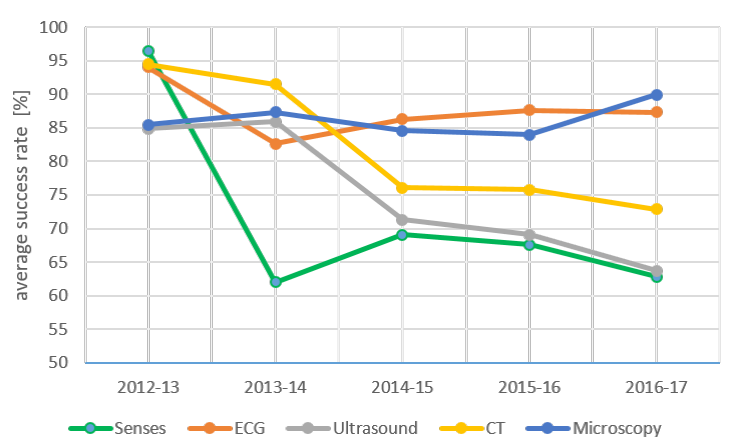

Fig. 1 Average success rate [\%] for Online tests for the last 5 years

The values displayed are always arithmetic means of the success rate in percentage, since this is what Moodle enables. Figure 1 shows that in the first academic year $(2012 / 13)$, online tests were not successful to the same degree. The main reason was that not all online tests were designed by the same author. In the following academic year, questions in the online tests were supposed to change to make tests more difficult. This manifested mostly in the Senses test and ECG test. A very small change occurred in the $C T$ test, which may not have necessarily been caused by the change of the questions. For the next academic year (2014/15), questions for the Ultrasound test and CT test were adjusted, based on the protocol in Moodle. This influenced success rates for both tests in that academic year. Based on the attached chart, we can make an observation that the authors succeeded in their attempts to unify difficulty levels of the Senses, Ultrasound, and CT tests from that academic year onwards. The general trend showing success rate dropping for the three tests mentioned above confirms this fact. And as the tests have not been updated since the 2014/15 academic year and the success rate drop is still obvious, we will have to focus on this drop if it manifests in the upcoming years. Last but not least, the chart clearly shows the Microscopy test and ECG test have been resulting in significantly higher success rates in the last 
three years than the remaining 3 tests. However, a lower level of difficulty of both of these topics might be the cause. Since each student has to finish all 5 online tests, it is not necessary to unify levels of all online tests.

From the general didactics point of view, topic 8 in the above described course is interesting. A Moodle database is created within this topic. Presentations on additional topics students choose and process in the work group mentioned above are uploaded in this database. Unlike submitting via email, this method's benefit is that a teacher can filter presentations based on fields created in the database and does not manage their receipt. Additionally, group reporting applies to the entire course; therefore, a teacher can select one of five study groups for verification. Moodle settings enable setting up separate groups, which means that, for example, students from the $2^{\text {nd }}$ group cannot see other groups' presentations. Having uploaded their presentations in Moodle within the defined period, each work group (1 through 10) within each of the five study groups delivers its presentation during Student Project Presentation. Only then is the student's activity named Student Project Submission in Moodle marked as Satisfactory.

Two continuous tests, Continuous Test - Statistics and Continuous Test - Biophysics, are prepared for students in the final course topic. Students complete both tests in the IT room across various days - per each study group, based on the schedule. The test usually includes the Calculated Question type. This task type enables the creation of various numerical values in the test instructions. Both tests are also restricted by a password, time lock, and IP address. Therefore, it is virtually impossible for students to complete the test at another time than within the pre-defined time period under an assistant's supervision. This format of continuous tests was established in the 2012/13 academic year; test settings have not changed, even though a new test is created every year. Again, Moodle provides success rate statistics for both tests for the last 5 years, see Fig. 2 and Tab. 2.

TABLE II.

AVERAGE SUCCESS RATE [\%] FOR BOTH CONTINUOUS TESTS

\begin{tabular}{|l|l|l|}
\hline & Test - statistics & \multicolumn{1}{c|}{ Test - biophysics } \\
\hline $\mathbf{2 0 1 2} / \mathbf{1 3}$ & 74,06 & 56,18 \\
\hline $\mathbf{2 0 1 3} / \mathbf{1 4}$ & 82,3 & 73,06 \\
\hline $\mathbf{2 0 1 4} / \mathbf{1 5}$ & 77,67 & 63,15 \\
\hline $\mathbf{2 0 1 5} / \mathbf{1 6}$ & 72,37 & 67,85 \\
\hline $\mathbf{2 0 1 6} / \mathbf{1 7}$ & 81,53 & 67,38 \\
\hline
\end{tabular}

No common trend seems to emerge out of the tests. The main reason lies in a different concept of both tests; the biophysics test almost exclusively uses the Calculated Question type. Variability of statistics-test tasks is significantly lower than that of biophysics. Moreover, the task pool used to select tasks is much broader for

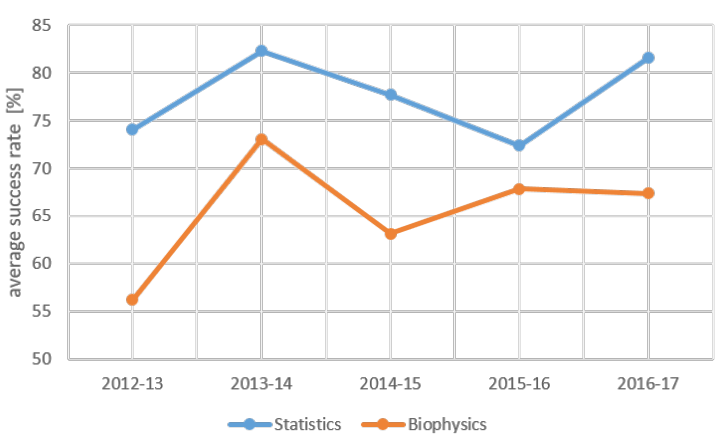

Fig. 2 Average success rate [\%] for both continuous tests

biophysics. These 2 factors result in the obvious conclusion you can see in Fig. 2: a higher success rate of the statistics test from the long-term perspective. Another factor that influences the rate is that the biophysics test should actually be more difficult, which was our intention, and it is confirmed by Fig. 2 . The change of the average success rate of the statistics test where the difference between the minimum and maximum is less than 10 (roughly 1.5 tasks) indicates fairly low fluctuation during 5 years and is no surprise to us. The test contains 15 tasks selected from 16 task categories where one task is selected from each category. Therefore, variability of test options is not very high, unlike the biophysics test where the type variability is significantly higher. Table 3 shows the higher variability.

TABLE III.

NUMBER OF CATEGORIES AND NUMBER OF TASKS FOR BOTH CONTINUOUS TEST

\begin{tabular}{|l|l|l|}
\hline \multicolumn{1}{|c|}{ Computer } & \multicolumn{1}{|c|}{$\begin{array}{c}\text { Number of } \\
\text { categories }\end{array}$} & \multicolumn{1}{c|}{$\begin{array}{c}\text { Number of } \\
\text { tasks }\end{array}$} \\
\hline $\begin{array}{l}\text { Test - } \\
\text { Biophysics }\end{array}$ & 20 & 65 \\
\hline Test - Statistics & 16 & 25 \\
\hline
\end{tabular}

One reason to create the above mentioned "multicourse" was a possibility to provide users with all information on the subject within a single course. At the same time, a course designed in this way provides teachers with a comprehensive overview of student grades. Teachers who create grade overviews can use filters to select grades of a particular study group from all 5 study groups. Most of the activities are graded automatically, except for the student project database, where the teacher has to save a grade named Student Project - Satisfactory for each student in the database, after the student has delivered their presentation as described above. Nevertheless, this manually entered grade also appears in the grade overview.

The grade overview of each student contains the following items: Database - Student Projects (the grade entered by the teacher), Senses test, ECG test, Ultrasound test, Microscopy test, CT test, Test 1 - Statistics, and Test 2 - Biophysics (grades applied automatically). The only item of all graded student activities, which is not included in this "multicourse", is Protocol Evaluation. As indicated above, 
the protocols are interactive and are evaluated automatically using Excel macros. When a protocol is submitted, metadata is saved to a student record in the $P C$ Doctor system. The protocol and student's record in PC Doctor are linked together using the student's ISIC card number the student enters to both the protocol and their record in PC Doctor. Therefore, the entire process is fully automated, except for the final verification stage which must be executed manually by the teacher.

\section{CONCLUSION}

We came to a conclusion that a targeted complex evaluation method brings benefits for both teachers and students. Students can browse points awarded in each activity throughout the entire term. Moreover, the evaluation is fully unbiased. The main advantage for teachers is in reduction of the time they used to need to correct tests. The main disadvantage of this complex evaluation is that, as of today, it is not possible to automatically evaluate interactive protocols we created and assign them to the Moodle system. Therefore, our current objective remains unchanged - to integrate protocols and LFHK Moodle so that the data of a correctly evaluated protocol is loaded to LFHK Moodle, assigned to the respective student, and added to the grade overview. This way, the grade overview of each student will be fully automated and complete. It will then make sense to create linked scoring and an overall grade of the course for each point-awarded activity. This method basically enables the award of a credit without being potentially "biased" by the teacher. In the time of publishing this article, this option had not been finished and, therefore, no conclusion from a complex evaluation system we head toward within the course can be presented.

\section{ACKNOWLEDGMENT}

We would like to thank to our IT support staff from the IS/IT Division of our faculty for their invaluable help.

\section{REFERENCES}

[1] J. Prucha, E. Walterova, J. Mares, "Pedagogicky slovnik," Praha: Portal, pp. 395, 2009, ISBN: 9788073676476.

[2] J. Feberova, T. Dostalova, M. Hladikova et al., "Evaluation of 5-year Experience with E-learning Techniques at Charles University in Prague. Impact on Quality of Teaching and Students' Achievements," New Educ. Rev., vol. 21, no. 2, pp. 110-120, 2010.

[3] M. Minovic, V. Stavljanin, M. Milovanovic et al., "Usability issues of e-learning systems: case-study for Moodle learning management system," On the Move to Meaningful Internet Systems: OTM 2008 Workshops, pp. 561-570, Nov. 2008. http://dx.doi.org/10.1007/978-3540-88875-8 79

[4] J. Hanus, T. Nosek, J. Zahora et al., "On-line integration of computer controlled diagnostic devices and medical information systems in undergraduate medical physics education for physicians," Phys. Medica, vol. 29, no. 1, pp. 83-90, Jan. 2013, http://dx.doi.org/10.1016/j.ejmp.2011.12.002.

[5] J. Hanus, J. Zahora, V. Masin et al., "On-Line Incorporation of Study and Medical Information System in Undergraduate Medical Education, "in 6th International Conference of Education, Research and Innovation (iceri 2013). Proceedings, Seville, Spain, 2013, pp. 1500-1507.

[6] J. Zahora, J. Hanus, D. Jezbera et al., "Remotely Controlled Laboratory and Virtual Experiments in Teaching Medical Biophysics," in 6th International Conference of Education, Research and Innovation (iceri 2013). Proceedings, Seville, Spain, 2013, pp. 900-906.

[7] J. Zahora, A. Bezrouk, J. Hanus, "Models of stents - Comparison and applications," Physiological Research, vol. 56, pp. 115-121, 2007.

[8] A. Bezrouk, L. Balsky, M. Smutny et al., "Thermomechanical properties of nickel-titanium closed-coil springs and their implications for clinical practice," Am. J. Orthod. Dentofac. Orthop., vol. 146, no. 3, pp. 319-327, $\quad$ Sep. 2014 http://dx.doi.org/10.1016/j.ajodo.2014.05.025.

[9] A. Bezrouk, L. Balsky, I. Selke Krulichova et al., "Nickel-titanium closed-coil springs: evaluation of the clinical plateau," Rev. Chim., vol. 68 , no. 5 , pp. 1137-1142, May 2017. 